\title{
Associations of symptomatic knee OA with histopathologic features in subchondral bone
}

\begin{tabular}{|c|c|}
\hline Journal: & Arthritis \& Rheumatology \\
\hline Manuscript ID & ar-18-0822.R2 \\
\hline Wiley - Manuscript type: & Full Length \\
\hline $\begin{array}{l}\text { Date Submitted by the } \\
\text { Author: }\end{array}$ & 07-Dec-2018 \\
\hline Complete List of Authors: & $\begin{array}{l}\text { Aso, Koji; University of Nottingham, Arthritis Research UK Pain Centre \& } \\
\text { NIHR Nottingham Biomedical Research Centre; Kochi Medical School, } \\
\text { Kochi University, Department of Orthopedic Surgery } \\
\text { Shahtaheri, Mohsen; University of Nottingham, Arthritis Research UK } \\
\text { Pain Centre \& NIHR Nottingham Biomedical Research Centre } \\
\text { Hill, Roger; Sherwood Forest Hospitals NHS Foundation Trust } \\
\text { Wilson, Deborah; Sherwood Forest Hospitals NHS Foundation Trust } \\
\text { McWilliams, Daniel; University of Nottingham, Arthritis Research UK Pain } \\
\text { Centre \& Division of ROD } \\
\text { Walsh, David; University of Nottingham, Arthritis Research UK Pain } \\
\text { Centre; Sherwood Forest Hospitals NHS Foundation Trust }\end{array}$ \\
\hline Keywords: & $\begin{array}{l}\text { Knee osteoarthritis, Subchondral bone, Pain, Nerve growth factor, } \\
\text { Osteoclast }\end{array}$ \\
\hline $\begin{array}{r}<\mathrm{B}>\text { Disease Category }</ \mathrm{b}>\text { : } \\
\text { Please select the category } \\
\text { from the list below that best } \\
\text { describes the content of your } \\
\text { manuscript.: }\end{array}$ & Osteoarthritis \\
\hline
\end{tabular}

\section{SCHOLARONE \\ Manuscripts}




\section{Running title; Associations of symptomatic knee OA with subchondral bone pathologies}

\section{Associations of symptomatic knee OA with histopathologic features in subchondral bone}

Koji Aso, MD, $\mathrm{PhD}^{1,3}$, Seyed M Shahtaheri, $\mathrm{PhD}^{1}$, Roger Hill ${ }^{1,2}$, Deborah Wilson, RGN ${ }^{1,2}$, Daniel F. McWilliams, $\mathrm{PhD}^{1}$, David A. Walsh, FRCP, PhD ${ }^{1,2}$.

1 Arthritis Research UK Pain Centre \& NIHR Nottingham Biomedical Research Centre, University of Nottingham, NG5 1PB, UK.

2 Sherwood Forest Hospitals NHS Foundation Trust, Mansfield

Road, Sutton in Ashfield, NG17 4JL, UK.

3 Department of Orthopedic Surgery, Kochi Medical School, Kochi University, 185-1

Oko-cho Kohasu, Nankoku 783-8505, Japan.

*Corresponding author: Koji Aso, MD PhD

Arthritis Research UK Pain Centre,

Clinical Sciences Building, City Hospital,

Nottingham, NG5 1PB

Tel: +44 (0) 1158231759

Fax: $+44(0) 1158231757$

ORCID: 0000-0003-3763-9564

\section{Funding sources}

This study was supported by a grant from by Arthritis Research UK (Centre initiative grant number 20777) and grant of Japanese Orthopaedic Society Knee, Arthroscopy and Sports Medicine, 2016.

\section{Conflict of interest}

D.A. Walsh: Grants from Arthritis Research UK, during the conduct of the study; grants from Pfizer Ltd, other from Pfizer Ltd, personal fees from GlaxoSmithKline, outside the submitted work.

Daniel F. McWilliams: grants from Pfizer Ltd.

The remaining authors have no conflicts of interest to declare. 


\section{Abstract \\ Objectives}

Subchondral bone and the osteochondral junction are thought to contribute to osteoarthritis (OA) knee pain. We aimed to identify osteochondral pathologies specifically associated with symptomatic human knee OA.

\section{Methods}

Two groups of medial tibial plateau ( $\mathrm{n}=31$ per group) were matched for macroscopic chondropathy scores. One group had undergone total knee replacement for OA knee pain (symptomatic chondropathy). The other had not sought help for knee pain and died from unrelated illness (asymptomatic chondropathy). OA histopathology, immunoreactivity for nerve growth factor (NGF) and CD68 (macrophages), tartrate resistant acid phosphatase (TRAP)-positive subchondral osteoclasts and synovitis were compared between groups.

\section{Results}

Mankin score, subchondral bone density and subchondral CD68-immunoreactive macrophage infiltration were similar between the 2 groups. NGF-like immunoreactivity was in subchondral mononuclear cells and osteoclasts, as well as in chondrocytes. NGF in osteochondral channels, and osteoclast densities in subchondral bone were higher in symptomatic than in asymptomatic chondropathy groups (NGF; $p<0.01$, TRAP; $p=0.02$ ), as also were synovitis scores $(p<0.01)$. Osteochondral pathology was not significantly associated with synovitis score. The differences in NGF expression and in osteoclast density remained significant after adjusting for age and synovitis score (NGF; $p=0.01$, TRAP; $p=0.04)$. Osteochondral NGF and osteoclast densities, together with synovitis scores, explained approximately $28 \%$ of sample allocation to symptomatic or asymptomatic groups.

\section{Conclusion}

Subchondral pathology was associated with symptomatic knee OA independently of chondropathy and synovitis. Increased NGF expression in osteochondral channels, and osteoclast density appear be key features associated with bone pain in knee OA. 


\section{Introduction}

Pain is the major source of disability and reason for hospital visits in patients with knee osteoarthritis (OA). Structural changes including articular cartilage degradation, synovial inflammation, osteophytes and subchondral osteosclerosis are characteristic of OA, but are not always accompanied by severe pain. Recent evidence suggests that subchondral bone contributes to knee OA pain ${ }^{1-7}$. Subchondral bone marrow lesions (BMLs) detected on magnetic resonance imaging (MRI) in knee OA are strongly associated with pain ${ }^{1-4,7}$. Bone attrition, a flattening or depression of the subchondral bone visualised using $\mathrm{x}$-rays or MRI, is also associated with the presence of pain ${ }^{5,6}$. Microarray analysis of BMLs in OA demonstrated upregulation of genes implicated in neurogenesis, osteochondral turnover and inflammation that might contribute to OA pain ${ }^{8}$. In animals, OA caused upregulation of nociceptive markers (calcitonin gene-related peptide and tropomyosin receptor kinase A (TrkA)) in subchondral bone afferents ${ }^{9}$. However, the mechanisms by which subchondral pathology contribute to OA pain are incompletely understood. Synovitis has also been associated with OA pain ${ }^{1,10-13}$. Synovial and subchondral pathology can occur together within the same joint, but it is unknown whether these represent discrete painful pathologies that could be separate targets for therapeutic intervention.

Nerve growth factor (NGF) plays a key role in the generation of acute and chronic pain, especially in inflammation ${ }^{14,15}$. NGF can bind two receptors: the high affinity $\operatorname{TrkA}^{16}$ and the low affinity p75 neurotrophin receptor ${ }^{17}$. NGF blockade can be achieved using antibodies or TrkA-IgG fusion protein that bind NGF and prevent its interaction with TrkA and p75 receptors. Recent clinical trials showed that NGF blockade remarkably reduced OA knee pain ${ }^{18,}{ }^{19}$. In human OA, NGF is upregulated in synovium ${ }^{10}$ and 
subchondral bone ${ }^{20}$. Increased synovial NGF expression was associated with symptomatic knee $\mathrm{OA}^{10}$, although the relevance of subchondral NGF expression has not been clarified. Increased density of tartrate resistant acid phosphatase (TRAP)-positive osteoclasts in subchondral bone was also associated with OA and knee symptoms ${ }^{21,22}$. Inflammatory CD68-positive macrophages were also detected in subchondral bone marrow compartments in human $\mathrm{OA}^{23}$.

We hypothesized that structural, cellular and molecular changes in subchondral bone are associated with symptomatic knee OA. We compared between case groups with similar macroscopic chondropathy but differing symptom severities. One group had sought help for knee pain and undergone total knee replacement (TKR) surgery (symptomatic chondropathy). The other group had not sought help for knee pain but had died from unrelated illness (asymptomatic chondropathy). We hypothesized that NGF expression by cells within subchondral bone was associated with symptomatic OA.

\section{Patients and Methods;}

\section{Patient samples}

Cases comprised 31 consecutive symptomatic chondropathy cases who had donated tibial plateau at TKR for OA and 31 asymptomatic chondropathy cases who had not presented with knee pain. All symptomatic chondropathy cases undertaking TKR report severe knee pain. All asymptomatic chondropathy cases had not sought medical attention for knee pain during the last year. The asymptomatic chondropathy cases are highly likely to have experienced less pain than the symptomatic chondropathy cases. Asymptomatic chondropathy cases were selected from 782 consecutive post-mortem (PM) donors by matching to each symptomatic chondropathy case for macroscopic chondropathy score 
and the percentage of joint surface with grade 4 chondropathy lesion [subchondral bone exposure] (each within \pm 5 between matched cases). Informed consent was obtained from TKR cases, or the next of kin of PM cases. Protocols were approved by Nottingham 1 Research Ethics Committee [05/Q2403/24] and Derby Research Ethics Committee 1 [11/H0405/2]. Symptomatic chondropathy samples were from patients fulfilling American College of Rheumatology classification criteria for $\mathrm{OA}^{24}$ at the time of TKR.

\section{Macroscopic chondropathy score and osteophytes}

Following tissue harvesting, articular surfaces of the medial tibial plateau were evaluated on the extent and severity of loss of surface integrity by a single assessor ${ }^{25}$. Articular surface defects were graded 0 [normal, smooth unbroken surface], 1 [swelling and softening], 2 [superficial fibrillation], 3 [deep fibrillation] and 4 [subchondral bone exposure]. The proportion of articular surface area corresponding to each grade was used to calculate a macroscopic chondropathy score (0-100) by the following formula Macroscopic chondropathy score $(0-100)=($ Grade $1 \times 0.14)+($ Grade $2 \times 0.34)+$ (Grade $3 \times 0.65)+$ Grade $4{ }^{25}$. Osteophytes were documented on direct visualization of PM samples as present or absent.

\section{Radiographic OA severity score.}

Radiographic OA severity scores were derived using preoperative postero-anterior knee radiographs as previously described ${ }^{25}$. An atlas of line drawings of the knee joint was used to grade medial and lateral joint space narrowing and osteophytes ${ }^{26}$. The scores for tibiofemoral joint space narrowing (range 0-6) and osteophytes (range 0-12) were summed to provide a total radiographic OA severity score (range $0-18)^{25}$.

\section{Sample processing}


143 Mid-coronal sections of the middle third of medial tibial plateaux (an important weight

144 bearing area characteristically affected by OA) were fixed in neutral buffered formalin

145 then decalcified in 10\% ethylenediaminetetraacetic acid (EDTA) in $10 \mathrm{mM}$ Tris buffer

$146\left(\mathrm{pH} 6.95,4^{\circ} \mathrm{C}\right)$ prior to wax embedding. Synovial tissues were fixed in formalin and

147 wax embedded without decalcification.

\section{Histology and grading}

149 Tibial plateaux sections $(5 \mu \mathrm{m})$ were stained with haematoxylin and eosin, or Safranin-

$150 \mathrm{O}$ and fast green. OA articular cartilage changes were graded using the Mankin scoring system $^{27}$; cartilage surface integrity (0 [normal] to 6 [complete disorganisation]), tidemark integrity $(0$ [intact] or 1 [crossed by vessels]), chondrocyte morphology $(0$ [normal] to 3 [hypocellular]) and proteoglycan loss (0 [normal, no loss of Safranin-O stain] to 4 [complete loss of stain]). Subchondral bone marrow replacement by fibrovascular tissue was assessed as either present or absent. Subchondral osteosclerosis was histologically assessed using trabecular bone volume per total volume (BV/TV) and subchondral plate area $(\mu \mathrm{m} 2 / \mu \mathrm{m})$; which were quantified using computer-assisted image analysis (Zeiss Systems). Osteochondral channel densities were assessed for subchondral bone, calcified cartilage and non-calcified cartilage separately in each region. Channels passing through one region into another were counted as in the region occupied by the larger part of the channel. Synovial inflammation was assessed using synovitis histological score developed by Haywood et al ${ }^{28}$; (0 [no synovitis] to 3 [severe synovitis]).

\section{Immunohistochemistry}

Sections underwent antigen retrieval $\left(10 \mathrm{mM}\right.$ citrate buffer, $\left.90^{\circ} \mathrm{C}, 20 \mathrm{mins}\right)$ and blocked with $5 \%$ bovine serum albumin (BSA) containing goat serum, followed by incubation 
with rabbit monoclonal antibody to NGF (EP1320Y, Abcam, Cambridge, UK), and biotinylated goat anti-rabbit IgG secondary antibody (BA1000, Vector, Peterborough, UK). CD68 immunoreactivity was visualized after citrate buffer antigen retrieval $\left(1 \mathrm{mg} / \mathrm{ml}\right.$ pepsin in $0.5 \mathrm{M}$ acetic acid, $\left.37^{\circ} \mathrm{C}, 2 \mathrm{~h}\right)$, and incubations with mouse monoclonal anti-human CD68 (MA5-13324, Thermo Fisher, MA, USA), and biotinylated horse anti-mouse IgG secondary antibody (BA2001, Vector, Peterborough, UK). Visualisation of NGF and CD68 immunoreactivites used avidin-biotincomplex (ABC) peroxidise (Vector, Peterborough, UK) with nickel-enhanced diaminobenzidine (DAB) development ${ }^{29}$. Sections were counterstained with hematoxylin so that different regions are more apparent.

NGF expression was measured as proportion (\%) of osteochondral channels in each case that displayed NGF-immunoreactive cells. Subchondral tissues within 400 micrometers of the cement line in the osteochondral junction were classified as bone marrow or fibrovascular tissues and NGF-like immunoreactivity was graded in each subchondral tissue type as: 0 , none; 1 , focal/sparse distribution; and 2 , high density, and in chondrocyte as: grades 0 ( $<5 \%$ of cells); 1 (5-20\% of cells); and $2(>20 \% \text { of cells })^{20}$.

183 CD68-immunoreactive macrophages were graded in subchondral tissues as: 0 , none; 1 , 184 focal/sparse distribution; and 2, high density ${ }^{20}$.

\section{Tartrate-Resistant Acid Phosphatase (TRAP) Staining}

Differentiated osteoclasts were identified by TRAP staining, using a commercially available kit (\#386A Sigma-Aldrich, $160 \mathrm{UK}$ ) following the manufacturer's protocol. TRAP positive osteoclasts were counted within $400 \mu \mathrm{m}$ of the cement line in the osteochondral junction and divided by the length of the subchondral bone to give an osteoclast density expressed as TRAP positive cells per $\mathrm{mm}^{22}$. One dark purplish or 
191

192

193

194

195

196

197

198

199

200

201

202

203

204

205

206

207

208

209

210

211

212

213

214

reddish cell with at least 3 nuclei or more was counted as one osteoclast.

\section{Image analysis}

All histological scoring and quantification was undertaken by a single observer (KA) who was blinded to diagnostic group, using a Zeiss Axioscop-50 microscope (Carl Zeiss, Welwyn Garden City, UK).

\section{Statistical analysis}

Statistical analyses were performed with JMP, Version 10 (SAS Ins. Cary, NC).

Comparisons used Mann-Whitney U or chi-square tests. Logistic regression was performed to adjust for age and synovitis scores and to calculate McFadden's pseudo$\mathrm{R}^{2}$. The $\mathrm{R} 2$ for each linear regression model was recorded for each of the individual histological measures (NGF alone, osteoclasts alone, or synovitis score alone) and also for the linear regression model where all measures were included together (NGF, osteoclasts and synovitis). Spearman's rank correlation (r) assessed associations. $P<0.05$ indicated statistically significance.

\section{Results}

\section{Patient details}

Demographics and sample details of cases selected for this study and for source repository cases are shown in Table 1. The selected asymptomatic chondropathy group had similar macroscopic chondropathy score and proportion of joint surface area displaying grade 4 chondropathy by matching to the symptomatic chondropathy group. The asymptomatic chondropathy group however had more severe OA changes than did the total cases in the post mortem repository from which they were selected. The asymptomatic chondropathy group was older than the symptomatic chondropathy group. There were no cases using 
medications for osteoporosis in either group.

Histological characteristics

Histological characteristics of the study groups are shown in Figure 1 and Table 2.

Osteochondral channels containing inflammatory cells and blood vessels were observed in subchondral bone plate, calcified cartilage and non-calcified cartilage (Figure 1A, B). Mankin score, proportion of cases with fibrovascular marrow replacement, histological $\mathrm{BV} / \mathrm{TV}$, subchondral plate area and osteochondral channel densities were similar between symptomatic and asymptomatic chondropathy groups. However, synovitis scores were higher in the symptomatic than in the asymptomatic chondropathy group, and this difference remained significant after adjusting for age $(\mathrm{aOR}=2.75$ [95\% CI 1.35-6.20], $p=0.01)$.

In samples of medial tibial plateau, NGF-immunoreactivity was detected in chondrocytes, subchondral mononuclear cells and in multinucleate osteoclast-like cells adherent to bone (Figure 1). NGF-immunoreactive cells were found in osteochondral channels, and in subchondral fibrovascular tissue and bone marrow (Figure 1). CD68immunoreactive macrophages were observed mainly in subchondral bone marrow and fibrovascular tissues (Figure 1). A higher proportion of osteochondral channels contained NGF-immunoreactive cells in the symptomatic than in the asymptomatic chondropathy group (Figure 2). This difference remained significant after adjusting for age and synovitis histological score $(\mathrm{aOR}=1.05$ [95\% CI 1.01-1.10], $p=0.01)$. Scores for subchondral macrophage infiltration, and NGF-immunoreactivity in chondrocytes and subchondral fibrovascular tissue and bone marrow did not differ significantly between groups (Supplementary table 1). NGF-immunoreactive osteochondral channels were significantly associated with Mankin score and with its component scores for tidemark 
integrity and cartilage surface integrity (Supplementary table 2). subchondral bone (Figure 1). The density of osteoclasts in the subchondral bone in the symptomatic chondropathy group was significantly higher than in the asymptomatic chondropathy group $(p=0.02)$ (Figure 2$)$. This difference remained significant after adjusting for age and synovitis score $(\mathrm{aOR}=1.19$ [95\% CI 1.01-1.48], $p=0.04)$. The percentage of NGF positive osteochondral channels was significantly correlated with the number of TRAP-positive osteoclasts $(\mathrm{r}=0.34, p=0.01)$. The association between NGF expression in osteochondral channels and symptomatic chondropathy remained significant after adjusting for osteoclasts density (aOR=1.05 [95\% CI 1.01-1.09], $p<0.01)$, but the significant association of osteoclast density with symptomatic chondropathy did not persist after adjusting for NGF expression in osteochondral channels $(\mathrm{aOR}=1.10$ [95\% confidence interval $0.96-1.32], p=0.20)$. Synovitis scores were not significantly associated with either NGF-immunoreactive osteochondral channels $(\mathrm{r}=0.07, p=0.62)$, nor with subchondral TRAP-positive osteoclasts $(\mathrm{r}=0.11, p=0.44)$. combination of all 3 histopathological features.

\section{Discussion}

We demonstrate components of subchondral pathology associated with symptomatic chondropathy in people undergoing knee arthroplasty for painful OA. We show that NGF expression in osteochondral channels and subchondral TRAP-positive osteoclast density each is associated with symptomatic chondropathy. We confirm previous findings ${ }^{10}$ that 
symptomatic $\mathrm{OA}$ is associated with synovitis, and show that associations with subchondral pathology are not dependent on the severity of chondropathy or synovitis. OA can affect all tissues in the joint, and our data support a model of OA pain to which different joint tissue compartments make discrete contributions.

\section{Associations of symptomatic knee OA with osteochondral NGF}

We found that the proportion of osteochondral channels positive for NGFimmunoreactivity was a sensitive measure able to distinguish symptomatic and asymptomatic case groups, supporting a role for osteochondral NGF in the generation of OA pain. This association appears to be over and above any effect of synovitis or cartilage damage on joint pain. The number of osteochondral channels penetrating into noncalcified cartilage is increased in $\mathrm{OA}^{20}$, but our findings suggest that this alone may not be sufficient to explain OA pain. We show that NGF-immunoreactivity in osteochondral channels was correlated with tidemark integrity, suggesting expression of sensitizing factors such as NGF as mediating effects of channels on OA pain.

NGF may directly activate sensory neurons that express TrkA and modulate the expression of TrkA or p75 receptor ${ }^{30}$. Anti-NGF antibodies can reduce OA pain ${ }^{18,} 19$ indicating the importance of NGF in pain generation, although their anatomical site of action remains uncertain. NGF has previously been localized to human synovium where it could be associated with OA pain ${ }^{10}$. OA chondrocytes may also express $\mathrm{NGF}^{10}$ although we were unable to demonstrate association of chondrocyte-derived NGF with symptomatic chondropathy. pain, by increasing colocalized sensory nerve activity. NGF immunoreactive cells were 
colocalized with sensory nerve fibers within osteochondral channels in human subchondral bone ${ }^{20}$. Indeed, most sensory neurons innervating the subchondral bone in rat knee joints were TrkA immunoreactive ${ }^{31}$, and TrkA expression in subchondral bone afferents was further increased during mono-iodoacetate-induced OA in rats ${ }^{9}$.

\section{Associations of symptomatic knee OA with osteoclasts}

293 Our results showed that osteoclast density in subchondral bone was associated with symptomatic knee OA and the differences remained significant after adjusting for age and synovitis histological score. Osteoclasts might increase pain either directly by changing the subchondral biochemical milieu, or by altering subchondral bone structure.

Osteoclasts release protons that generate a local acidosis, potent activators of nociceptors that can increase pain signaling ${ }^{32}$. Our findings also indicate that osteoclasts are a source of NGF which could then sensitise primary afferents in the subchondral bone. NGF-immunoreactivity, but not by subchondral trabecular bone density. Our current results therefore extend findings from a previous study ${ }^{22}$ which reported a potential role of increased osteoclast density in subchondral bone in the generation of OA pain. High serum concentration of TRAP5b, an indicator of osteoclast number, was associated with subchondral osteoclast density, OA pain and worse pain prognosis ${ }^{22}$. We now show that association of osteoclast density with symptomatic OA is not explained by associations with chondropathy, synovitis, or age, suggesting a direct effect of osteoclasts on OA pain. Increased subchondral osteoclast number was also associated with pain behavior in rats ${ }^{33}$, ${ }^{34}$, and reducing the number of osteoclasts led to decreases in weight bearing pain ${ }^{34}$. Studies of osteoclast inhibitors such as bisphosphonates, denosumab and strontium 
311 ranelate show reductions joint pain in people with knee $\mathrm{OA}^{35}{ }^{36}$. The bisphosphonate

312 zoledronic acid reduced knee pain and BML size in people with $\mathrm{OA}^{36}$, although a metaanalysis of randomized controlled trials did not support analgesic effects of bisphosphonates in knee $\mathrm{OA}^{37}$. Our data suggest that OA knee pain has multiple sources, and targeting osteoclasts will only have clinically important benefit in those cases where osteoclast activity is the predominant driver of pain.

Associations between NGF and osteoclasts

We show associations between NGF and osteoclast densities in subchondral bone.

Multinucleated osteoclasts were immunoreactive for NGF, and NGF expression in osteochondral channels was significantly correlated with the number of TRAP-positive osteoclasts. NGF expression in osteochondral channels was associated with symptomatic knee OA after adjusting for osteoclast density, but association of osteoclasts density with symptoms did not persist after adjusting for NGF. Our data support the view that NGF is a more important factor than osteoclast density in subchondral bone for the generation of OA pain.

Furthermore, NGF can act as an autocrine or paracrine factor regulating osteoclast activity and bone remodeling. NGF and TrkA are expressed by osteoclasts, and the addition of NGF to monocyte cultures induces the formation of TRAP-positive multinucleated cells ${ }^{38}$. An anti-NGF antibody reduced subchondral osteoclast numbers in a rat model of OA pain ${ }^{39}$. 
changes in discrete tissue compartments of the human knee. Cases with more severe chondropathy are more likely to display synovitis and subchondral bone changes ${ }^{40}$. However, in the current study, subchondral changes were not significantly associated with synovitis grade and each compartment might contribute discretely to OA pain.

Our findings support a heterogeneous model of OA pain, resulting from multiple mechanisms in different peripheral tissues. The balance between pain mechanisms varies from person to person. Latent class analysis has indicated that synovitis is a key characteristic defining one subgroup of people with $\mathrm{OA}^{10}$. Our findings here suggest that subchondral pathology can define a subgroup of people with symptomatic chondropathy, only partially overlapping with cases whose OA pain is driven by synovitis.

MRI evidence of cartilage defects ${ }^{41}$, bone marrow lesions ${ }^{7}$ and synovitis ${ }^{12}$ can also discretely predict OA pain. We extend these findings to identify NGF-immunoreactive osteochondral channels and subchondral osteoclast densities as key pathological features which make discrete contributions to OA symptoms.

Our results showed that $28 \%$ of group allocation to symptomatic and asymptomtic chondropathy can be explained by the combination of synovitis score, NGF expression in osteochondral channels and subchondral osteoclast density. Synovitis score and NGF expression in osteochondral channels contributed to group allocation to similar extents (17\% and $13 \%$, respectively), and both may be important targets for future OA treatments.

\section{Limitations}

This study has several potential limitations. Some patients in our 'asymptomatic' chondropathy group might have experienced knee pain, but relatives may have been unaware of these symptoms. However, all patients undertaking TKR report severe knee pain, and it is highly likely that people who have not undergone surgery overall have less 
pain than those who do. Symptomatic and asymptomatic chondropathy groups differed by age, although significant associations with subchondral pathology and synovitis persisted after adjusting our analyses for age. Samples were from the mid-coronal section of the medial tibial plateau, a key weight bearing area, but findings might differ for other joint regions such as femoral condyles. Symptomatic chondropathy cases had late-stage OA undergoing arthroplasty, and different pain mechanisms might be important in cases with less severe structural change. Osteoclast activity itself was not examined in this study. However, cell with at least 3 nuclei or more was counted as one osteoclast to estimate active osteoclasts, as resorption activity has been shown under some circumstances to correlate with the number of nuclei ${ }^{42}$. However, osteoclast numbers do not necessarily correlate with osteoclast activity, for example during bisphosponate treatment ${ }^{43}$. More direct measures, for example of biomarkers of collagen breakdown, might further clarify whether associations of symptoms with osteoclast number might reflect mediation by osteoclast activity. Our models did not explain all of the variance in classification to symptomatic and asymptomatic groups. Some variation might be attributable to case ascertainment (e.g. people in the asymptomatic group might have experienced some knee pain). Factors not explored here, such as other histopathologic changes, cytokines/molecules, psychological factors, biomechanical loading and obesity, are likely to also contribute to OA pain. BMLs are associated with knee OA pain. BMLs have been associated with cartilage surface integrity and subchondral bone marrow replacement by fibrovascular tissue ${ }^{8}$, both of which were similar between symptomatic and asymptomatic chondropathy groups in our study. However, MRI scans were not available for cases in our study, and further investigation is needed to clarify the association of BMLs with NGF expression in osteochondral channels and TRAP-positive osteoclast densities. Case 
matching asymptomatic chondropathy cases from a total post-mortem sample group of 782 knees enabled us to identify histopathological factors contributing to OA symptoms, but further research would need determine their importance relative to contributions from chondropathy itself.

\section{Conclusions}

We have identified histopathologic features of subchondral bone that are associated with symptomatic chondropathy. NGF expression in osteochondral channels was associated with symptomatic knee OA over and above any effects of chondropathy, synovitis and subchondral TRAP-positive osteoclast densities. Increased NGF expression appears as a key features associated with subchondral bone pain in knee OA, and could contribute to the previously observed association between osteoclasts and OA pain. Our data support a heterogeneous model of OA pain, with discrete contributions from different compartments in the joint. Different treatments could benefit pain from synovitis or from subchondral pathology, necessitating the development of biomarkers to help target treatments to those who will most benefit. Other treatments targeting molecular pathways that are shared between tissue compartments will have greater potential for efficacy in unselected OA populations.

\section{Acknowledgements}

We express our sincere gratitude to all patients who participated in this study.

\section{Author Contributions}

All authors approved the final version to be published. K.A. had full access to all of the data in the study and takes responsibility for the integrity of the data and the accuracy of the data analysis. K.A., D.M. and D.W. designed the experiments, analyzed and interpreted results, and wrote the manuscript. K.A. and M.S. did immunohistochemistry, histological analysis. K.A., D.M. and D.W. analyzed and interpreted the results. 


\section{Ethics approval}

411 Nottingham 1 Research Ethics Committee [05/Q2403/24] and Derby Research Ethics 412 Committee $1[11 / \mathrm{H} 0405 / 2]$.

\section{Provenance and peer review}

414 Not commissioned; externally peer reviewed.

\section{References}

417 1. Zhang Y, Nevitt M, Niu J, Lewis C, Torner J, Guermazi A et al. Fluctuation of knee

5. Hernandez-Molina G, Neogi T, Hunter DJ, Niu J, Guermazi A, Reichenbach S et al.

6. Reichenbach S, Dieppe PA, Nuesch E, Williams S, Villiger PM, Juni P. Association

7. Lo GH, McAlindon TE, Niu J, Zhang Y, Beals C, Dabrowski C et al. Bone marrow lesions and joint effusion are strongly and independently associated with weightbearing pain in knee osteoarthritis: Data from the osteoarthritis initiative. Osteoarthritis Cartilage 2009;17:1562-9.

8. Kuttapitiya A, Assi L, Laing K, Hing C, Mitchell P, Whitley G et al. Microarray analysis of bone marrow lesions in osteoarthritis demonstrates upregulation of genes implicated in osteochondral turnover, neurogenesis and inflammation. Ann Rheum Dis 2017;76:1764-73. phenotype alterations of dorsal root ganglia neurons innervating the subchondral 
bone in osteoarthritic rat knee joints. Osteoarthritis Cartilage 2016;24:1596-603.

10. Stoppiello LA, Mapp PI, Wilson D, Hill R, Scammell BE, Walsh DA. Structural associations of symptomatic knee osteoarthritis. Arthritis Rheumatol 2014;66:301827.

11. Sarmanova A, Hall M, Fernandes GS, Bhattacharya A, Valdes AM, Walsh DA et al. Association between ultrasound-detected synovitis and knee pain: A populationbased case-control study with both cross-sectional and follow-up data. Arthritis Res Ther 2017;19:281.

12. Wang X, Jin X, Han W, Cao Y, Halliday A, Blizzard L et al. Cross-sectional and longitudinal associations between knee joint effusion synovitis and knee pain in older adults. J Rheumatol 2016;43:121-30.

13. Hill CL, Hunter DJ, Niu J, Clancy M, Guermazi A, Genant H et al. Synovitis detected on magnetic resonance imaging and its relation to pain and cartilage loss in knee osteoarthritis. Ann Rheum Dis 2007;66:1599-603.

14. Watson JJ, Allen SJ, Dawbarn D. Targeting nerve growth factor in pain: What is the therapeutic potential? BioDrugs 2008;22:349-59.

15. Barthel C, Yeremenko N, Jacobs R, Schmidt RE, Bernateck M, Zeidler H et al. Nerve growth factor and receptor expression in rheumatoid arthritis and spondyloarthritis. Arthritis Res Ther 2009;11:R82.

16. Kaplan DR, Hempstead BL, Martin-Zanca D, Chao MV, Parada LF. The trk protooncogene product: A signal transducing receptor for nerve growth factor. Science 1991;252:554-8.

17. Mallett S, Barclay AN. A new superfamily of cell surface proteins related to the nerve growth factor receptor. Immunol Today 1991;12:220-3.

18. Lane NE, Schnitzer TJ, Birbara CA, Mokhtarani M, Shelton DL, Smith MD et al. Tanezumab for the treatment of pain from osteoarthritis of the knee. N Engl J Med 2010;363:1521-31.

19. Sanga P, Katz N, Polverejan E, Wang S, Kelly KM, Haeussler J et al. Efficacy, safety, and tolerability of fulranumab, an anti-nerve growth factor antibody, in the treatment of patients with moderate to severe osteoarthritis pain. Pain 2013;154:1910-9.

20. Walsh DA, McWilliams DF, Turley MJ, Dixon MR, Franses RE, Mapp PI et al. Angiogenesis and nerve growth factor at the osteochondral junction in rheumatoid arthritis and osteoarthritis. Rheumatology (Oxford) 2010;49:1852-61.

21. Prieto-Potin I, Largo R, Roman-Blas JA, Herrero-Beaumont G, Walsh DA. Characterization of multinucleated giant cells in synovium and subchondral bone in 
482

483

484

485

486

487

488

489

490

491

492

493

494

495

496

497

498

499

500

501

502

503

504

505

506

507

508

509

510

511

512

513

514

515

516

517

knee osteoarthritis and rheumatoid arthritis. BMC Musculoskelet Disord 2015;16:226.

22. Nwosu LN, Allen M, Wyatt L, Huebner JL, Chapman V, Walsh DA et al. Pain prediction by serum biomarkers of bone turnover in people with knee osteoarthritis: An observational study of tracp5b and cathepsin $\mathrm{k}$ in oa. Osteoarthritis Cartilage 2017;25:858-65.

23. Geurts J, Patel A, Hirschmann MT, Pagenstert GI, Muller-Gerbl M, Valderrabano $\mathrm{V}$ et al. Elevated marrow inflammatory cells and osteoclasts in subchondral osteosclerosis in human knee osteoarthritis. J Orthop Res 2016;34:262-9.

24. Altman R, Asch E, Bloch D, Bole G, Borenstein D, Brandt K et al. Development of criteria for the classification and reporting of osteoarthritis. Classification of osteoarthritis of the knee. Diagnostic and therapeutic criteria committee of the american rheumatism association. Arthritis Rheum 1986;29:1039-49.

25. Walsh DA, Yousef A, McWilliams DF, Hill R, Hargin E, Wilson D. Evaluation of a photographic chondropathy score (pcs) for pathological samples in a study of inflammation in tibiofemoral osteoarthritis. Osteoarthritis Cartilage 2009;17:30412.

26. Nagaosa Y, Mateus M, Hassan B, Lanyon P, Doherty M. Development of a logically devised line drawing atlas for grading of knee osteoarthritis. Ann Rheum Dis 2000;59:587-95.

27. Mankin HJ, Dorfman H, Lippiello L, Zarins A. Biochemical and metabolic abnormalities in articular cartilage from osteo-arthritic human hips. Ii. Correlation of morphology with biochemical and metabolic data. J Bone Joint Surg Am 1971;53:523-37.

28. Haywood L, McWilliams DF, Pearson CI, Gill SE, Ganesan A, Wilson D et al. Inflammation and angiogenesis in osteoarthritis. Arthritis Rheum 2003;48:2173-7.

29. Shu SY, Ju G, Fan LZ. The glucose oxidase-dab-nickel method in peroxidase histochemistry of the nervous system. Neurosci Lett 1988;85:169-71.

30. Pezet S, McMahon SB. Neurotrophins: Mediators and modulators of pain. Annu Rev Neurosci 2006;29:507-38.

31. Aso K, Ikeuchi M, Izumi M, Sugimura N, Kato T, Ushida T et al. Nociceptive phenotype of dorsal root ganglia neurons innervating the subchondral bone in rat knee joints. Eur J Pain 2014;18:174-81.

32. Jimenez-Andrade JM, Mantyh WG, Bloom AP, Ferng AS, Geffre CP, Mantyh PW. Bone cancer pain. Ann N Y Acad Sci 2010;1198:173-81.

33. Strassle BW, Mark L, Leventhal L, Piesla MJ, Jian Li X, Kennedy JD et al. 
Inhibition of osteoclasts prevents cartilage loss and pain in a rat model of degenerative joint disease. Osteoarthritis Cartilage 2010;18:1319-28.

34. Sagar DR, Ashraf S, Xu L, Burston JJ, Menhinick MR, Poulter CL et al. Osteoprotegerin reduces the development of pain behaviour and joint pathology in a model of osteoarthritis. Ann Rheum Dis 2014;73:1558-65.

35. Reginster JY, Badurski J, Bellamy N, Bensen W, Chapurlat R, Chevalier X et al. Efficacy and safety of strontium ranelate in the treatment of knee osteoarthritis: Results of a double-blind, randomised placebo-controlled trial. Ann Rheum Dis 2013;72:179-86.

36. Laslett LL, Dore DA, Quinn SJ, Boon P, Ryan E, Winzenberg TM et al. Zoledronic acid reduces knee pain and bone marrow lesions over 1 year: A randomised controlled trial. Ann Rheum Dis 2012;71:1322-8.

37. Vaysbrot EE, Osani MC, Musetti MC, McAlindon TE, Bannuru RR. Are bisphosphonates efficacious in knee osteoarthritis? A meta-analysis of randomized controlled trials. Osteoarthritis Cartilage 2018;26:154-64.

38. Hemingway F, Taylor R, Knowles HJ, Athanasou NA. Rankl-independent human osteoclast formation with april, baff, ngf, igf i and igf ii. Bone 2011;48:938-44.

39. Xu L, Nwosu LN, Burston JJ, Millns PJ, Sagar DR, Mapp PI et al. The anti-ngf antibody mumab 911 both prevents and reverses pain behaviour and subchondral osteoclast numbers in a rat model of osteoarthritis pain. Osteoarthritis Cartilage 2016;24:1587-95.

40. Niu J, Felson DT, Neogi T, Nevitt MC, Guermazi A, Roemer F et al. Patterns of coexisting lesions detected on magnetic resonance imaging and relationship to incident knee osteoarthritis: The multicenter osteoarthritis study. Arthritis Rheumatol 2015;67:3158-65.

41. Moisio K, Eckstein F, Chmiel JS, Guermazi A, Prasad P, Almagor O et al. Denuded subchondral bone and knee pain in persons with knee osteoarthritis. Arthritis Rheum 2009;60:3703-10.

42. Boissy P, Saltel F, Bouniol C, Jurdic P, Machuca-Gayet I. Transcriptional activity of nuclei in multinucleated osteoclasts and its modulation by calcitonin. Endocrinology 2002;143:1913-21.

43. Weinstein RS, Roberson PK, Manolagas SC. Giant osteoclast formation and longterm oral bisphosphonate therapy. N Engl J Med 2009;360:53-62.

\section{Figure 1: Histopathologic features in subchondral bone}

A; NGF-positive osteochondral channel (arrow head) in symptomatic chondropathy. B; 
554 NGF-negative osteochondral channel (arrow) in asymptomatic chondropathy. NGF555 immunoreactive cells (brown) were found in osteochondral channels (A), fibrovascular 556 tissue (C) and bone marrow (D). Multinucleated osteoclasts were immunoreactive for 557 NGF. (E). CD68-immunoreactive macrophages were mainly observed in bone marrow 558 (F) and fibrovascular tissue (G). TRAP staining showed multinucleated osteoclasts 559 (purple) $(\mathrm{H})$. Scale bars $=50 \mu \mathrm{m}$

560

561 Figure 2: Immunoreactivity for NGF and TRAP-positive osteoclasts in the 562 subchondral bone from symptomatic and asymptomatic chondropathy cases

563 Scatterplots illustrate the differences between symptomatic and asymptomatic 564 chondropathy. Lines represent medians and IQR. ${ }^{*} p<0.01$, and $\# p=0.02$ versus 565 asymptomatic chondropathy. 


\begin{tabular}{|c|c|c|c|}
\hline & $\begin{array}{l}\text { Symptomatic } \\
\text { chondropathy } \\
(n=31 \text { knees) }\end{array}$ & $\begin{array}{l}\text { Asymptomatic } \\
\text { chondropathy } \\
\text { (n=31 knees) }\end{array}$ & $\begin{array}{c}\text { Post-mortem } \\
\text { repository } \\
\text { (n= } 782 \text { knees) }\end{array}$ \\
\hline Macroscopic chondropathy score $(0-100)$ & $74(56,80)$ & $76(56,81) \#$ & $33(24,51)$ \\
\hline Joint surface area with grade 4 chondropathy (\%) & $30(0,48) *$ & $30(0,50)^{\# \#}$ & $0(0,0)$ \\
\hline Gender, Male (\%) & 51.6 & 61.3 & 54.5 \\
\hline Age (year) & $67(55,73) *$ & $74(66,84)^{\#}$ & $69(60,80)$ \\
\hline Total radiographic OA severity score $(0-18)$ & $13(10.5,13.5)$ & NA & NA \\
\hline Tibiofemoral JSN score (0-6) & $5(5,5.8)$ & NA & NA \\
\hline Medial tibiofemoral JSN score (0-3) & $3(3,3)$ & NA & NA \\
\hline Osteophyte score $(0-12)$ & $8(5.5,8)$ & NA & NA \\
\hline Medial tibial osteophyte score $(0-3)$ & $2(2,2)$ & NA & NA \\
\hline MFC osteophytes (Yes/No) & NA & $16 / 14(53.3 \%))^{\#}$ & $113 / 738(15.3 \%)$ \\
\hline LFC osteophytes (Yes/No) & NA & $18 / 11(62.1 \%)^{\# \#}$ & $111 / 738(15.0 \%)$ \\
\hline MT osteophytes (Yes/No) & NA & $15 / 15(50.0 \%) \# \#$ & $87 / 738(11.7 \%)$ \\
\hline LT osteophytes (Yes/No) & NA & $13 / 17(43.3 \%))^{\# \#}$ & $82 / 738(11.1 \%)$ \\
\hline Patellar osteophytes (Yes/No) & NA & $10 / 20(50.0 \%))^{\# \#}$ & $41 / 358(11.4 \%)$ \\
\hline
\end{tabular}

\section{Table 1: Patient and sample details}

Data displayed as median (IQR). Total radiographic OA severity score is a summation of tibiofemoral joint space narrowing (JSN) and osteophyte scores. Tibiofemoral JSN score is a summation of medial and lateral tibiofemoral JSN scores. Osteophyte score is a summation of medial and lateral tibial and femoral osteophyte scores. ${ }^{*} p<0.01$ versus asymptomatic chondropathy, $\# \mathrm{p}=0.03$, and $\# \# \mathrm{p}<0.01$ versus the post-mortem repository. JSN; joint space narrowing, MFC; medial femoral condyle, LFC; lateral femoral condyle, MT; medial tibial plateau, LT; lateral tibial plateau, NA = Not available.

\begin{tabular}{cccc}
\hline & $\begin{array}{c}\text { Symptomatic } \\
\text { chondropathy } \\
(\mathbf{n = 3 1} \text { knees) }\end{array}$ & $\begin{array}{c}\text { Asymptomatic } \\
\text { chondropathy } \\
(\mathbf{n}=\mathbf{3 1} \text { knees) }\end{array}$ & P \\
\hline Total Mankin score $(0-14)$ & $9(7,11)$ & $8(7,11)$ & 0.70 \\
Cartilage surface integrity $(0-6)$ & $4(3,6)$ & $4(3,6)$ & 0.98
\end{tabular}




\begin{tabular}{lccc}
\multicolumn{1}{c}{ Chondrocyte appearance $(0-3)$} & $2(2,3)$ & $2(2,2)$ & 0.45 \\
\multicolumn{1}{c}{ Tidemark integrity $(0-1)$} & $1(0,1)$ & $0(0,1)$ & 0.13 \\
\multicolumn{1}{c}{ Proteoglycan loss $(0-4)$} & $2(2,3)$ & $2(2,3)$ & 0.87 \\
Subchondral bone marrow replacement & $11 / 20(35 \%)$ & $14 / 17(45 \%)$ & 0.44 \\
(Yes/No) & & $57.3(39.0,63.0)$ & 0.95 \\
Histological BV/TV & $50.0(42.0,61.3)$ & $651.5(431.7,1050.0)$ & 0.43 \\
Subchondral plate area $\left(\mu \mathrm{m}^{2} / \mu \mathrm{m}\right)$ & $608.3(460.0,810.6)$ & $4.9(3.5,7.4)$ & 0.93 \\
Total osteochondral channel density & $5.4(3.7,6.4)$ & & 0.93 \\
$(/ \mathrm{mm})$ & & $4.7(3.4,7.2)$ & 0.51 \\
$\quad$ Subchodral bone $(/ \mathrm{mm})$ & $4.8(3.3,6.1)$ & $0.25(0,0.46)$ & 0.89 \\
Calcified cartilage $(/ \mathrm{mm})$ & $0.24(0.09,57)$ & $0(0,0)$ & $<0.01$ \\
\hline Non-calcified cartilage $(/ \mathrm{mm})$ & $0(0,0)$ & $1(1,2.5)$ & \\
Synovitis histological score $(0-3)$ & $3(2.75,3)$ & & \\
\hline
\end{tabular}

\section{Table 2: Osteochondral histology and synovitis scores}

Data displayed as median (IQR). Total Mankin score is a summation of cartilage surface integrity, chondrocyte appearance, tidemark integrity, and proteoglycan loss. BV/TV is trabecular bone volume per total volume. Total osteochondral channel density is a summation of osteochondral channel densities in subchondral bone, calcified cartilage and non-calcified cartilage. 

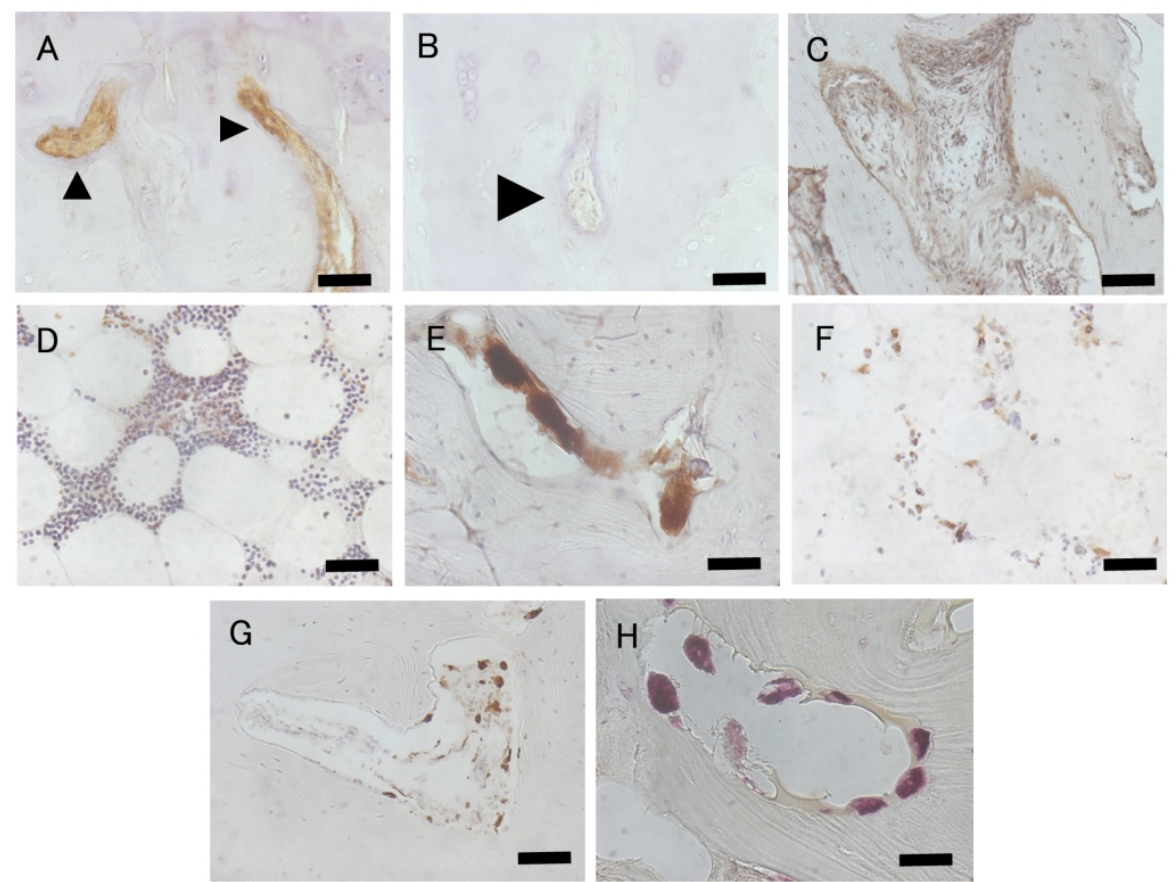

Figure 1: Histopathologic features in subchondral bone

A; NGF-positive osteochondral channel (arrow head) in symptomatic chondropathy. B; NGF-negative osteochondral channel (arrow) in asymptomatic chondropathy. NGF- immunoreactive cells (brown) were found in osteochondral channels (A), fibrovascular tissue (C) and bone marrow (D). Multinucleated osteoclasts were immunoreactive for NGF. (E). CD68-immunoreactive macrophages were mainly observed in bone marrow $(F)$ and fibrovascular tissue $(G)$. TRAP staining showed multinucleated osteoclasts (purple)

$(\mathrm{H})$. Scale bars $=50 \mu \mathrm{m}$

$175 \times 124 \mathrm{~mm}(300 \times 300$ DPI $)$ 

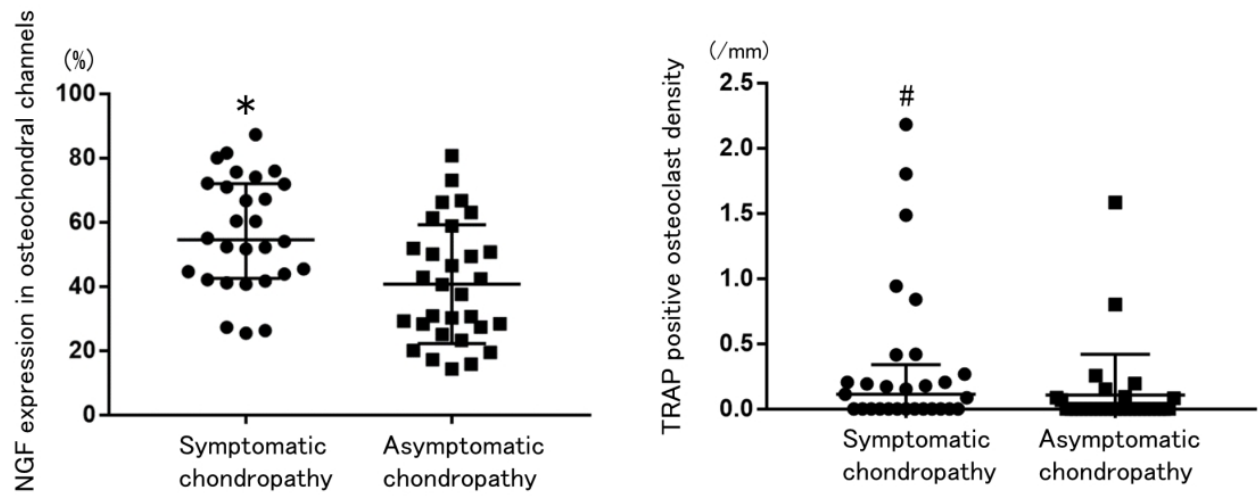

Figure 2: Immunoreactivity for NGF and TRAP-positive osteoclasts in the subchondral bone from symptomatic and asymptomatic chondropathy cases

Scatterplots illustrate the differences between symptomatic and asymptomatic chondropathy. Lines represent medians and IQR. ${ }^{*} p<0.01$, and $\# p=0.02$ versus asymptomatic chondropathy.

$170 \times 70 \mathrm{~mm}(300 \times 300 \mathrm{DPI})$ 


\begin{tabular}{|c|c|c|c|}
\hline & $\begin{array}{l}\text { Symptomatic } \\
\text { chondropathy } \\
\text { (n=31 knees) }\end{array}$ & $\begin{array}{l}\text { Asymptomatic } \\
\text { chondropathy } \\
\text { (n=31 knees) }\end{array}$ & $P$ \\
\hline NGF expression in fibrovascular tissue $(0-2)$ & $1(1,2)$ & $2(1,2)$ & 0.63 \\
\hline NGF expression in bone marrow $(0-2)$ & $1(0,1)$ & $1(0.75,2)$ & 0.11 \\
\hline NGF expression in chondrocyte $(0-2)$ & $1(1,2)$ & $1(1,2)$ & 0.70 \\
\hline $\begin{array}{l}\text { CD68-immunoreactive macrophage in } \\
\text { fibrovascular tissue }(0-2)\end{array}$ & $1(1,2)$ & $2(0.5,2)$ & 0.53 \\
\hline $\begin{array}{l}\text { CD68-immunoreactive macrophage in bone } \\
\text { marrow }(0-2)\end{array}$ & $1(1,1)$ & $1(0,2)$ & 0.67 \\
\hline
\end{tabular}

Supplementary table 1: Immunoreactivity for NGF and CD68 (macrophages) in the subchondral bone from symptomatic and asymptomatic chondropathy cases

Data displayed as median (IQR).

\begin{tabular}{|c|c|c|c|c|c|c|c|c|c|c|}
\hline & & \multicolumn{4}{|c|}{ NGF expression } & \multicolumn{5}{|c|}{ Mankin score } \\
\hline & & $\begin{array}{l}\text { Osteochon } \\
\text { dral } \\
\text { channels }\end{array}$ & $\begin{array}{c}\text { Fibrovascu } \\
\text { lar tissue }\end{array}$ & $\begin{array}{c}\text { Bone } \\
\text { marro } \\
\text { w }\end{array}$ & $\begin{array}{c}\text { Chondro } \\
\text { cytes }\end{array}$ & $\begin{array}{l}\text { Total } \\
\text { Mankin } \\
\text { score }\end{array}$ & $\begin{array}{l}\text { Cartilage } \\
\text { surface } \\
\text { integrity }\end{array}$ & $\begin{array}{l}\text { Chondrocyte } \\
\text { appearance }\end{array}$ & $\begin{array}{l}\text { Tidemark } \\
\text { integrity }\end{array}$ & $\begin{array}{c}\text { Proteogl } \\
\text { ycan } \\
\text { loss }\end{array}$ \\
\hline \multirow{4}{*}{ 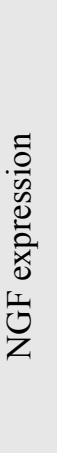 } & $\begin{array}{l}\text { Osteochondral } \\
\text { channels }\end{array}$ & 1 & 0.38 & 0.12 & 0.18 & $0.32 *$ & $0.26^{*}$ & 0.22 & $0.36 * *$ & 0.14 \\
\hline & $\begin{array}{l}\text { Fibrovascular } \\
\text { tissue }\end{array}$ & - & 1 & 0.30 & $0.49 *$ & $0.46^{*}$ & 0.21 & 0.03 & 0.23 & $0.52 *$ \\
\hline & Bone marrow & - & - & 1 & 0.17 & $0.29 *$ & 0.25 & 0.17 & 0.07 & 0.23 \\
\hline & Chondrocytes & - & - & - & 1 & 0.24 & 0.25 & 0.04 & 0.17 & 0.24 \\
\hline
\end{tabular}

Supplementary table 2: Correlation of NGF expression in subchondral bone tissue and chondrocytes with Mankin score

Data displayed as Spearman's r. ${ }^{*} p<0.05, * * p<0.01$ 